(2) Open Access Full Text Article

\title{
Investigation of biofilm formation on a charged intravenous catheter relative to that on a similar but uncharged catheter
}

This article was published in the following Dove Press journal:

Medical Devices: Evidence and Research

20 June 2014

Number of times this article has been viewed

\author{
Guy A Richards ${ }^{1,2}$ \\ Adrian J Brink ${ }^{3}$ \\ Ross Mclntosh ${ }^{4}$ \\ Helen C Steel ${ }^{5,6}$ \\ Riana Cockeran ${ }^{5,6}$ \\ 'Department of Critical Care, \\ Charlotte Maxeke Johannesburg \\ Academic Hospital, ${ }^{2}$ Faculty of \\ Health Sciences, University of the \\ Witwatersrand, Johannesburg, \\ South Africa; ${ }^{3}$ Department of \\ Clinical Microbiology, Ampath \\ National Laboratory Services, \\ Milpark Hospital, Johannesburg, \\ South Africa; ${ }^{4}$ Nano-Scale Transport \\ Physics Laboratory, School of Physics, \\ University of the Witwatersrand, \\ Johannesburg, South Africa; \\ ${ }^{5}$ Medical Research Council Unit \\ for Inflammation and Immunity, \\ Department of Immunology, \\ University of Pretoria, Pretoria, \\ South Africa; ${ }^{6}$ National Health \\ Laboratory Service, Pretoria, \\ South Africa
}

\begin{abstract}
Catheter-related blood stream infections increase morbidity, mortality, and costs. This study investigated whether Certofix ${ }^{\circledR}$ protect antimicrobial catheters carry a surface charge and whether this inhibits biofilm formation. The capacitance of the catheter surfaces was measured and, to determine if the catheters released ions, distilled water was passed through and current measured as a function of voltage. With probes touching the inner and outer surfaces, capacitance was not voltage-dependent, indicating surfaces were uncharged or carried a similar charge. When one probe penetrated the catheter wall, capacitance was weakly voltage-dependent, indicating the presence of a surface charge. Standard and charged catheters were also exposed to phosphate buffered saline as controls or $2 \times 10^{6}$ colony forming units $/ \mathrm{mL}$ (in phosphate buffered saline) of six different microorganisms for 60 or 120 minutes. When the growth of detached bacteria was measured, biofilm formation was significantly reduced, $(P<0.05)$, for charged catheters for all organisms.
\end{abstract}

Keywords: central venous catheters, electrical charge, biofilm

\section{Introduction}

Central venous catheters (CVCs) are essential in the treatment of critically ill patients. However, this increases the risk of catheter-related bloodstream infections (CRBSI), which increase morbidity, mortality, and costs. It has been estimated that 250,000-500,000 infections related to CVCs occur in the United States annually, adding $\$ 33,000-65,000$ per case to health care costs. ${ }^{1}$

There are many potential risk factors for CRBSI. These include, inter alia: longer duration of catheterization; the location of the CVC; the use of parenteral nutrition or multilumen CVCs; catheter care; and aseptic insertion technique. ${ }^{2}$

Several recent studies have investigated preventative strategies; these include the routine replacement of intravenous catheters, ${ }^{2,3}$ the development of "CVC bundles" or guidelines designed to reduce catheter-related infection (CRI), ${ }^{4}$ and antimicrobial, silver ion or antiseptic impregnated CVCs designed to prevent attachment of organisms. The latter has not conclusively demonstrated benefit, however. ${ }^{2,5}$ Implant-associated infections are caused by microorganisms that are adherent to the device surface and embedded in a complex, extracellular, three-dimensional, polymeric matrix called biofilm. Biofilm- associated infections are generally persistent, as the embedded organisms exchange mediators of resistance ${ }^{6}$ and evade eradication by the immune system, detergents and antimicrobials. ${ }^{6-8}$

Passerini et $\mathrm{al}^{9}$ have shown that $81 \%$ of all vascular catheters which had been in situ for between 1-14 days were colonized by bacteria in biofilm. These confer 10-1,000-fold
Correspondence: Guy A Richards Department of Medicine, Wits Medical School, 7 York Road, Parktown 2195, Johannesburg, South Africa

Tel +27 II 4883654

Fax +27 II 4884675

Email guy.richards@wits.ac.za 
greater resistance to antibiotics and microbiocides, when compared to their planktonic counterparts. ${ }^{6,10,11}$ Under unfavorable conditions such as overcrowding and nutrient deprivation, planktonic bacteria detach from the biofilm and enter the bloodstream causing bacteremia and colonization of new sites. ${ }^{12}$ Single or multiple bacterial species can make up a biofilm, with a dental biofilm containing more than 500 different bacterial taxa. ${ }^{13}$

In this regard, B Braun Melsungen AG (Melsungen, Germany) has recently developed a CVC (Certofix ${ }^{\circledR}$ protect antimicrobial catheter; catheter A) that is claimed to prevent biofilm formation by means of a surface charge generated by positively charged chemical structures on the internal and

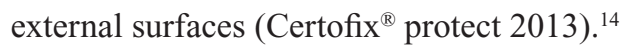

\section{Aims}

The aims of this study were to investigate whether the charge on the catheter actually exists and whether it inhibits microbial adherence or biofilm formation when using six different microorganisms on two CVCs, one charged and one uncharged, but similar in appearance.

\section{Materials and methods Investigation of catheter charge}

\section{Catheters}

The charged catheters were sourced from B Braun Melsungen AG and consisted of two components, a hydrophilic polymer, blended with polyurethane, and a polyhexanide functionalized with a methacrylate group (a biguanide salt). These two compounds have opposite charges and - as such - an ionic bond occurs between them with net polarization of the biguanide due to charge deficiency. This reportedly forms a polarized surface that releases ions extremely slowly. The other catheter was a standard central venous catheter, identical in appearance, manufactured from polyurethane and without charge or antiseptic, silver ion, or antibiotic impregnation. In the biofilm experiments, the investigators were blinded as to which catheter was which.

\section{Investigation of polarization}

To investigate the polarization of the exterior surface of the catheters, the capacitance between the inner and outer surfaces was measured as a function of voltage. Capacitors store electric charge, generally between two charged plates and dielectric materials, such as polyurethane, and can enhance capacitance. A fully discharged capacitor maintains zero volts across its terminals; whereas, one that is charged can maintain a potential across its terminals. Excess charge, in this case due to polarization of the catheter surface, can be detected through capacitance versus voltage measurements. The capacitive response of the catheters was measured at room temperature under atmospheric conditions as a function of applied voltage using a Janis micromanipulated probe station (Janis Research Company, LLC (Woburn, MA, USA) and an Agilent B1500A semiconductor device analyzer (Agilent Technologies, Santa Clara, CA, USA). The measurements were performed with one of the probes penetrating the surface of the catheter, while the other was in contact with the surface to determine if any surface charge was present.

Finally, to determine if the catheter released ions, distilled water was passed through the catheters and multiple measurements of current, as a function of voltage, were made and compared with that generated through distilled water alone.

\section{Estimation of effect on biofilm}

\section{Microorganisms utilized}

Staphylococcus aureus, S. epidermidis, Pseudomonas aeruginosa, Acinetobacter baumannii, Klebsiella pneumoniae, and Candida albicans were grown overnight to the mid-log phase, at $37^{\circ} \mathrm{C}$ in an atmosphere of $5 \% \mathrm{CO}_{2}$ in tryptic soy broth ([TSB]; Merck Millipore, Billerica, MA, USA), followed by turbidimetric numerical standardization of the culture, representing $2 \times 10^{6}$ colony forming units (cfu) $/ \mathrm{mL}$, in phosphate buffered saline ([PBS] $0.15 \mathrm{M}$; BD Diagnostics, Sparks Glencoe, MD, USA).

\section{Chemicals and reagents}

All chemicals and reagents, unless otherwise indicated, were obtained from Sigma-Aldrich Co (St Louis, MO, USA).

\section{Biofilm formation on intravenous catheters}

The catheters were cut into $15 \mathrm{~mm}$ sections and exposed to either PBS (untreated control) or $2 \times 10^{6} \mathrm{cfu} / \mathrm{mL}$ bacteria (in PBS) for 60 or 120 minutes at $37^{\circ} \mathrm{C}, 5 \% \mathrm{CO}_{2}$. Following incubation, the nonadherent bacteria were removed by washing the catheter sections with PBS. The washed sections were placed in TSB and incubated for 24 hours at $37^{\circ} \mathrm{C}, 5 \% \mathrm{CO}_{2}$. Following incubation, the growth of the detached bacteria was determined through both turbidity measurements at $540 \mathrm{~nm}$ using the PowerWave ${ }^{\mathrm{TM}}$ plate reader (Bio-Tek Instruments Inc., Winooski, VT, USA) and standard cfu enumeration procedures. Before the amount of biofilm could be determined, the nonadherent bacteria and 
medium were removed from the catheter sections by washing the sections three times with PBS. Following the removal of all the unbound bacteria, the catheter sections were stained with $0.1 \%$ crystal violet, and the excess dye was removed by washing with PBS. The crystal violet ${ }^{15}$ was released from the adherent bacteria by the addition of $96 \%$ ethanol and the amount of biofilm formed correlated directly with the color intensity of the crystal violet, which was determined spectrophotometrically at a wavelength of $570 \mathrm{~nm}$, using the PowerWave $\mathrm{X}^{\mathrm{TM}}$ plate reader.

\section{Statistical analysis}

Two to three experiments comprising 18-36 replicates in each system for each microorganism were performed. The results are expressed as mean \pm standard error of the mean. Statistical analysis was performed using the Mann-Whitney $U$ test (unpaired, nonparametric, two-tailed). ${ }^{*} P$ values of $\leq 0.05$ were considered significant.

\section{Results}

\section{Investigation of catheter charge}

Assessment of polarization

When the probes touched the inner and outer surfaces lightly, the capacitance was found to be voltage-independent (Figure 1). This indicates that the inner and outer surfaces of the capacitor are either uncharged or have the same charge. In addition, the capacitance was enhanced by the fact that the catheters are dielectric; ie, each surface is insulated from the other.

When one of the probes was inserted into the catheter wall, penetrating the outer surface, capacitance was found to be weakly voltage-dependent (Figure 2). As the potential was varied from negative to positive, the charge on the probes changed. This could only occur if one of the probes was in

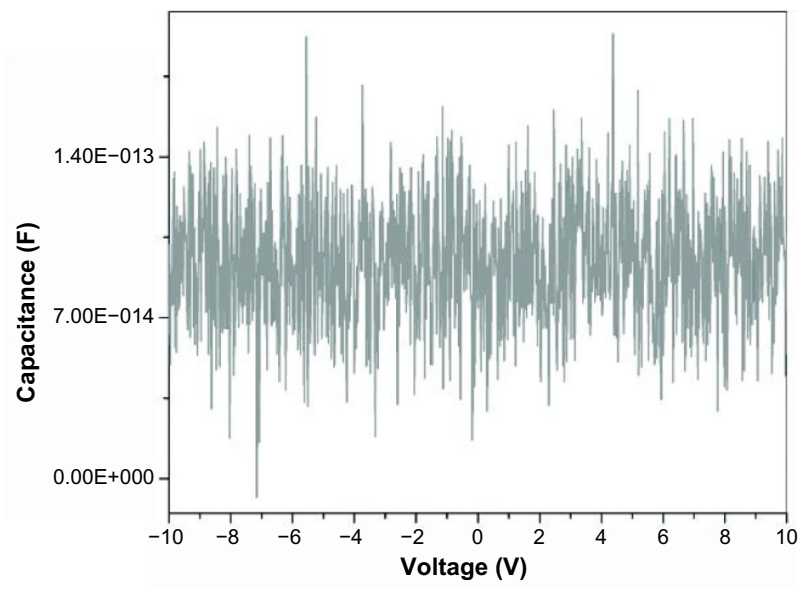

Figure I Capacitance versus voltage when both probes are touching the outer surface of the capacitor.

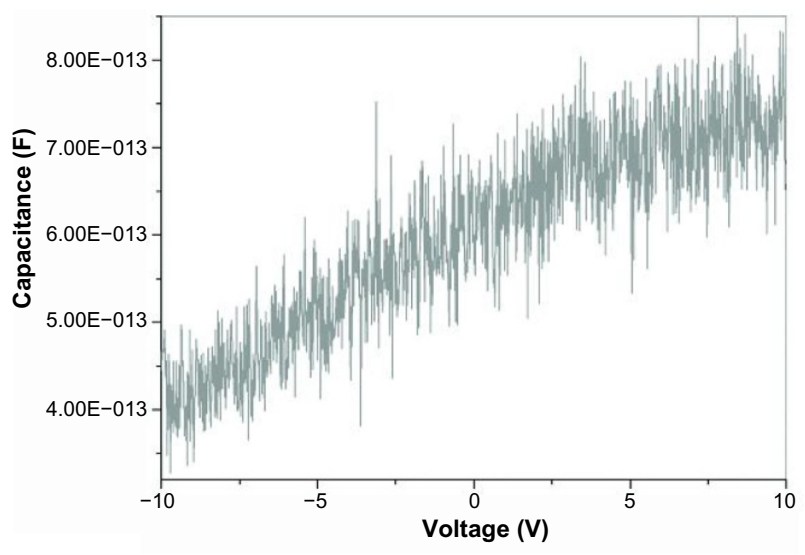

Figure 2 Capacitance versus voltage with one of the probes penetrating the catheter surface while the other is in contact with the surface.

contact with a charge, indicating that in this case, the outer surface is polarized. Also, since - as has been described previously - the capacitance is independent of voltage when both probes are in contact with the surface of the capacitor (Figure 1), the inner and outer surfaces of the capacitor must have the same charge. This was confirmed by the fact that when the potential across the two probes was reversed, the capacitance decreased slightly with voltage as would be expected since the excess charge is in contact with the opposite probe in this case (not shown).

\section{Assessment of release of ions}

In control samples consisting of distilled water, the current was measured as a function of voltage for $55 \mu \mathrm{L}$ droplets. Current is directly related to conductivity, so the presence of ions can be detected by measuring current as a function of voltage. The same measurements were then performed when distilled water was passed through the catheters, and a voltage applied. At any given voltage, the current (representative of conductivity) was smaller in the droplets of water that had been passed through the CVCs, indicating that ions had been adsorbed onto the catheter surface with a consequent decrease in conductivity. No direct evidence of release of ions was found; however, after the passage of greater volumes of distilled water through the catheter, the results were closer to those of the controls, indicating that the catheter surface had been saturated by ions acquired from the distilled water.

\section{Biofilm formation}

The amount of biofilm, after the untreated control has been subtracted, for each organism still adherent to the catheters is shown in the panel A series of Figures 3-7. The results for S. aureus and S. epidermidis were similar, and only those for 

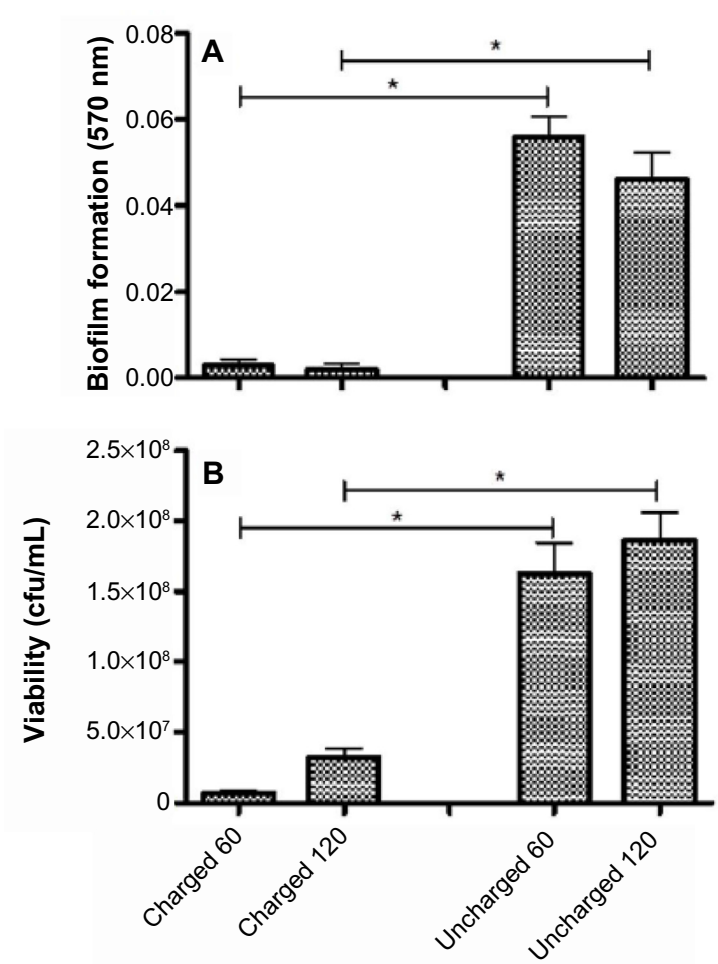

Figure 3 Charged and uncharged catheter sections were exposed to Staphylococcus aureus for 60 or 120 minutes. The amount of biofilm formed (A), and the amount of bacteria that detached from the biofilm in 24 hours measured as cfu/mL (B), are shown as the mean \pm SEM. Two to three experiments with between 18 and 36 replicates for each system were analyzed.

Note: $* p<0.05$.

Abbreviations: cfu, colony forming units; SEM, standard error of the mean.
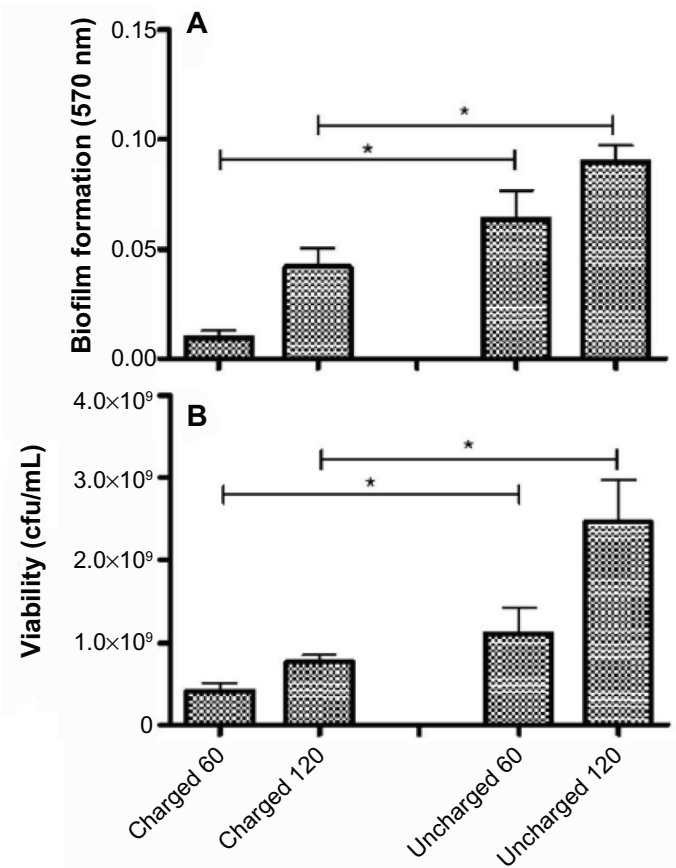

Figure 4 Charged and uncharged catheter sections were exposed to Pseudomonas aeruginosa for 60 or 120 minutes. The amount of biofilm formed (A), and the amount of bacteria that detached from the biofilm in 24 hours measured as $\mathrm{cfu} / \mathrm{mL}$ (B), are shown as the mean \pm SEM. Two to three experiments with between 18 and 36 replicates for each system were analyzed.

Note: $* P<0.05$.

Abbreviations: cfu, colony forming units; SEM, standard error of the mean.

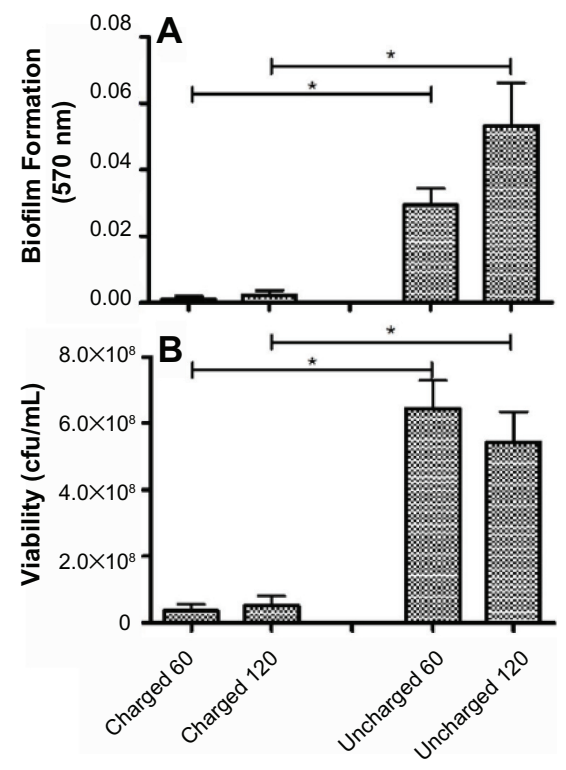

Figure 5 Charged and uncharged catheter sections were exposed to Klebsiella pneumoniae for 60 or 120 minutes. The amount of biofilm formed (A), and the amount of bacteria that detached from the biofilm in 24 hours measured as $\mathrm{cfu} / \mathrm{mL}$ (B), are shown as the mean \pm SEM. Two to three experiments with between 18 and 36 replicates for each system were analyzed.

Note: $* P<0.05$.

Abbreviations: cfu, colony forming units; SEM, standard error of the mean.
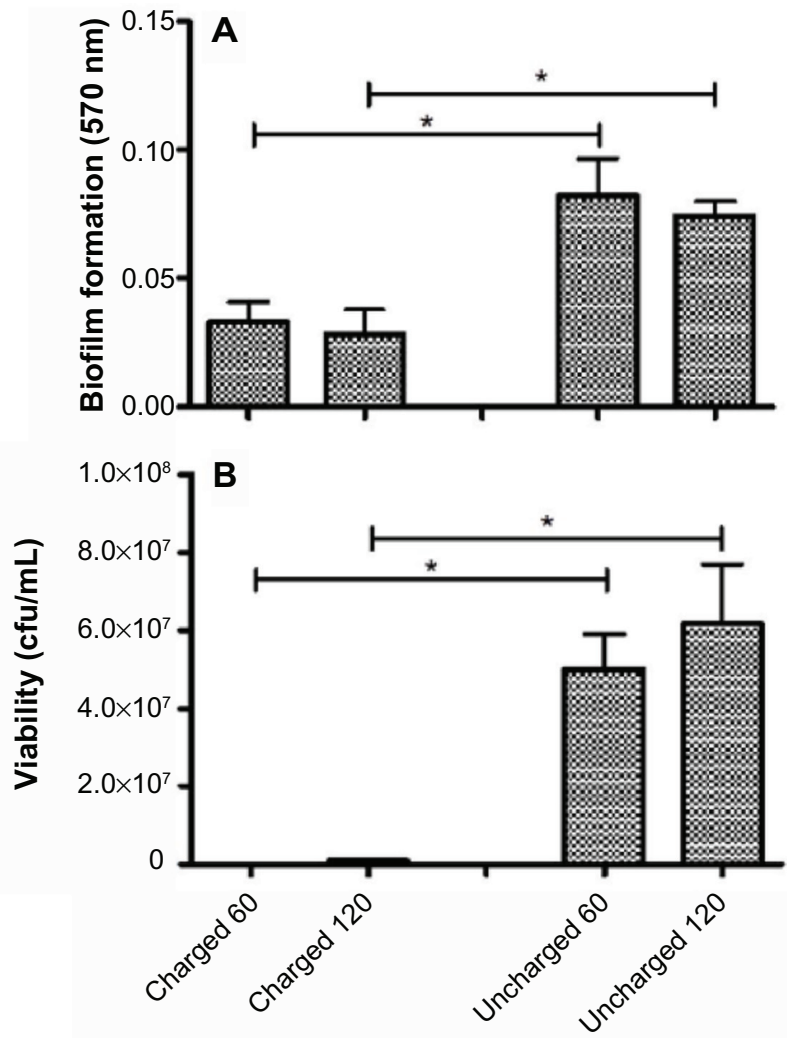

Figure 6 Charged and uncharged catheter sections were exposed to Acinetobacter baumanii for 60 or 120 minutes. The amount of biofilm formed (A), and the amount of bacteria that detached from the biofilm in 24 hours measured as cfu/mL (B), are shown as the mean \pm SEM. Two to three experiments with between 18 and 36 replicates for each system were analyzed.

Note: $* P<0.05$

Abbreviations: cfu, colony forming units; SEM, standard error of the mean. 


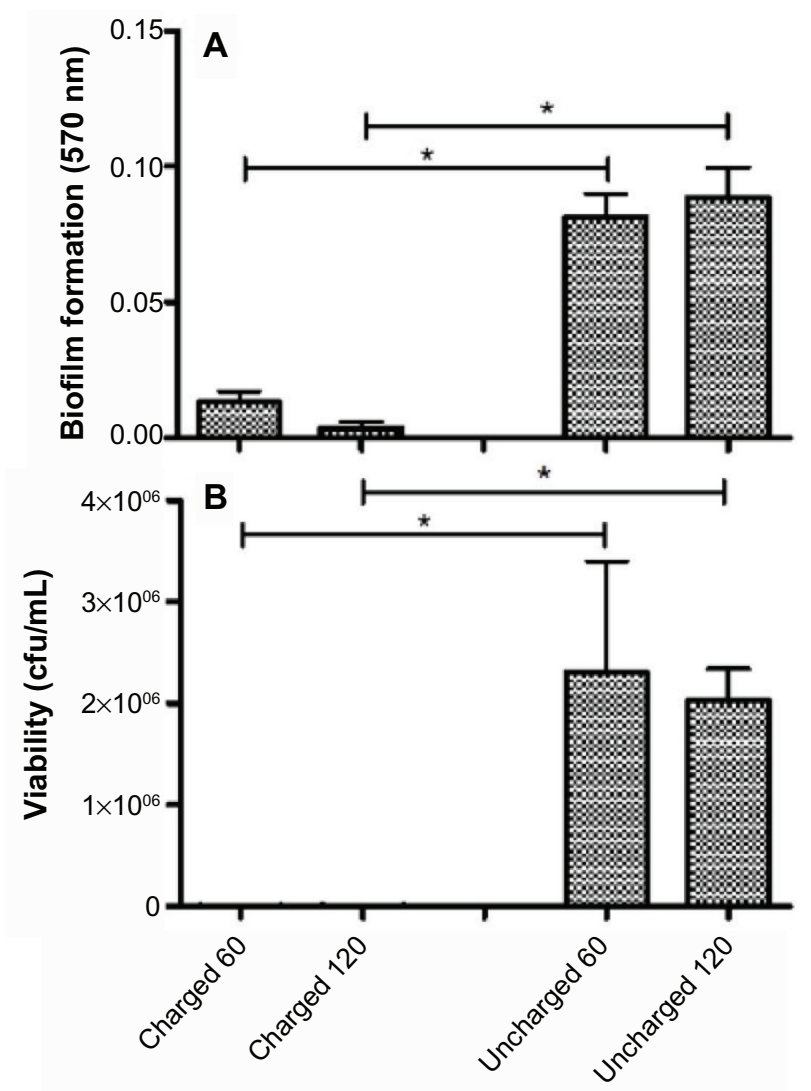

Figure 7 Charged and uncharged catheter sections were exposed to Candida albicans for 60 or 120 minutes. The amount of biofilm formed (A), and the amount of bacteria that detached from the biofilm in 24 hours measured as cfu/mL (B), are shown as the mean \pm SEM. Two to three experiments with between 18 and 36 replicates for each system were analyzed.

Note: $* P<0.05$.

Abbreviations: cfu, colony forming units; SEM, standard error of the mean.

S. aureus are shown. It is evident that there is a statistically significant difference $(P<0.05)$ between both the 60 - and 120-minute exposure times for all six organisms, with the charged catheter forming statistically less biofilm than the uncharged catheter at both time points. The quantity of bacteria that detached from the biofilm was determined by turbidity and $\mathrm{cfu} / \mathrm{mL}$, and as both of these growth measurements showed similar results, only the cfu/mL measurements were shown as depicted in the panel B series of Figures 3-7, each of which is representative of a different microorganism. Significantly fewer bacteria were present in the supernatant of the charged catheters at both time points, indicating fewer detached cells. It is noteworthy that the growth of P. aeruginosa in the uncharged catheter sections was greatly underestimated as detached bacteria proliferated to the extent that they showed visible clumping, which could not be broken up for fear of disrupting biofilm formation. As a consequence, fewer bacteria were considered for the growth determinations. Irrespective of this limitation, the difference between the two catheters still reached significance.

\section{Discussion}

Intravascular catheters are a major source of bloodstream infections in hospitalized patients. The prevalence of catheter-related infections is approximately five episodes per 1,000 catheter days in Europe ${ }^{16}$ and one to six episodes per 1,000 catheter days in the United States. ${ }^{1}$ These infections often result in increased morbidity and mortality and increase costs considerably. ${ }^{17}$ Microbial adherence is essential to the formation of biofilm; ${ }^{18}$ however, this adherence is dependent on the ionic interaction between the microorganism and the surface. ${ }^{19}$ It has been shown that a negative charge attracts bacteria, ${ }^{20}$ suggesting that a positively charged surface will repel bacteria and, thereby, inhibit adhesion. This principle has recently been explored with the use of the copper coating of commonly touched surfaces in the ICU, which significantly reduces hospital-acquired infection. ${ }^{21}$

This study investigated whether the Certofix ${ }^{\circledR}$ protect (charged) catheter had a polarized inner and outer surface as claimed, and whether adherence of microorganisms would be inhibited in comparison to an otherwise identical uncharged catheter.

We showed that both the external as well as the internal surfaces of the Certofix ${ }^{\circledR}$ protect catheter retained a small positive charge, which was maintained by ionic bonding and that no ions were released, indicating a stable product, which will retain its charge over an extended time period. This charge appeared to have a significant inhibitory effect on biofilm formation as well as decreasing the number of detached bacteria as determined by growth (optical density and cfu measurements), when compared to the uncharged catheters.

These differences were evident following 60- and 120-minute exposure times for all microorganisms tested. Even though this time period is short when compared to the time an intravenous catheter will be used, the catheters were exposed to a far higher concentration of microorganisms $\left(2 \times 10^{6}\right.$ cells $\left./ \mathrm{mL}\right)$ than is likely to occur in the clinical setting.

An additional limitation of the experimental design was the cutting of catheter sections. Even though great care was taken, the surface of the catheter could have been damaged, which could have provided a nidus for attachment, growth, and biofilm formation. Unfortunately, there was no feasible alternative method by which these experiments could have been performed and, in addition, both catheters were sectioned in an identical manner, and - despite this significant differences between the catheters persisted. The experimental design also catered only for total (surface and intraluminal) adherence and biofilm formation, and we could not distinguish between the two. 
It is possible that the absorbance of the ions from water, as well as the cutting of the catheters, may have had an impact on the charged catheter's ability to prevent adherence of bacteria over longer periods of time; however, a welldesigned, controlled clinical study should be able to answer this question. Several care facilities have introduced the utilization of bundles of care to reduce catheter-associated infections. Nonetheless, any additional intervention that could further impact on the morbidity and mortality associated with catheter use is to be welcomed.

\section{Acknowledgments}

B Braun Melsungen AG (Melsungen, Germany) supplied the catheters and sufficient funds to perform the biofilm studies in the form of an unrestricted grant. RC is supported by the National Research Foundation of South Africa. RM is supported by the National Research Foundation (SA) under the Nanotechnology Flagship Project grant and the University Research Council (Witwatersrand).

\section{Disclosure}

The authors report no conflicts of interest in this work.

\section{References}

1. Brooks JL, Jefferson KK. Staphylococcal biofilms: quest for the magic bullet. Adv Appl Microbiol. 2012;81:63-87.

2. Mer M, Duse AG, Galpin JS, Richards GA. Central venous catheterization: a prospective, randomized double-blind study. Clin Appl Thromb Hemost. 2009;15(1):19-26.

3. Rickard CM, Webster J, Wallis MC, et al. Routine versus clinically indicated replacement of peripheral intravenous catheters: a randomised controlled equivalence trial. Lancet. 2012;380(9847):1066-1074.

4. O'Grady NP, Alexander M, Dellinger EP, et al. Guidelines for the prevention of intravascular catheter-related infections. Centers for Disease Control and Prevention. MMWR Recomm Rep. 2002;51(RR-10):1-29.

5. McConnell SA, Gubbins PO, Anaissie EJ. Are antimicrobial-impregnated catheters effective? Replace the water and grab your washcloth, because we have a baby to wash. Clin Infect Dis. 2004;39(12):1829-1833.

6. Aslam S. Effect of antibacterials on biofilms. Am J Infect Control. 2008;36(10):S175. e9-S175. e11.
7. Fux CA, Costerton JW, Stewart PS, Stoodley P. Survival strategies of infectious biofilms. Trends Microbiol. 2005;13(1):34-40.

8. Hall-Stoodley L, Costerton JW, Stoodley P. Bacterial biofilms: from the natural environment to infectious diseases. Nat Rev Microbiol. 2004;2(2): 95-108.

9. Passerini L, Lam K, Costerton JW, King EG. Biofilms on indwelling vascular catheters. Crit Care Med. 1992;20(5):665-673.

10. Moscoso M, García E, López R. Pneumococcal biofilms. Int Microbiol. 2009;12(2):77-85.

11. Gilbert P, Maira-Litran T, McBain AJ, Rickard AH, Whyte FW. The physiology and collective recalcitrance of microbial biofilm communities. Adv Microb Physiol. 2002;46:202-256.

12. Tejedor SC, Tong D, Stein J, et al. Temporary central venous catheter utilization patterns in a large tertiary care centre: tracking the "idle central venous catheter". Infect Control Hosp Epidemiol. 2012;33(1): $50-57$.

13. Suresh GK, Edwards WH. Central line-associated bloodstream infections in neonatal intensive care: changing the mental model from inevitability to preventability. Am J Perinatol. 2012;29(1):57-64.

14. Certofix ${ }^{\circledR}$ protect - Antipathogenic central venous catheter (CPAC) for long-term protection against catheter infection [webpage on the Internet]. German Healthcare Export Group. Available from: http:// www.gheg.de/en/products/product/4575_Certofix_protect_Antipathogenic_central_venous_catheter_for_long-term_protection_against_ catheter_infection/index.html. Accessed January 6, 2014.

15. Vesterlund S, Paltta J, Karp M, Ouwehand AC. Measurement of bacterial adhesion-in vitro evaluation of different methods. J Microbiol Methods. 2005;60(2):225-233.

16. van Eck van der Sluijs A, Oosterheert JJ, Ekkelenkamp MB, Hoepelman IM, Peters EJ. Bacteremic complications of intravascular catheter tip colonization with Gram-negative micro-organisms in patients without preceding bacteremia. Eur J Clin Microbiol Infect Dis. 2012;31(6):1027-1033.

17. Dimick JB, Pelz RK, Consunji R, Swoboda SM, Hendrix CW, Lipsett PA. Increased resource use associated with catheter-related bloodstream infection in the surgical intensive care unit. Arch Surg. 2001;136(2):229-234.

18. Zhang L, Gowardman J, Rickard CM. Impact of microbial attachment on intravascular catheter-related infections. Int J Antimicrob Agents. 2011;38(1):9-15.

19. Toba FA, Visai L, Trivedi S, Lowy FD. The role of ionic interactions in the adherence of the Staphylococcus epidermidis adhesion SdrF to prosthetic material. FEMS Microbiol Lett. 2013;388(1):24-30.

20. Yamashita Y, Kunimori A, Takehara T. Effect of calcium ions on cell surface electrostatics of Bacteroides gingivalis and other oral bacteria. Zentralbl Bakteriol. 1991;275(1):46-53.

21. Salgado CD, Sepkowitz KA, John JF, et al. Copper surfaces reduce the rate of healthcare-acquired infections in the intensive care unit. Infect Control Hosp Epidemiol. 2013;34(5):479-486.
Medical Devices: Evidence and Research

\section{Publish your work in this journal}

Medical Devices: Evidence and Research is an international, peerreviewed, open access journal that focuses on the evidence, technology, research, and expert opinion supporting the use and application of medical devices in the diagnosis, treatment and management of clinical conditions and physiological processes. The identification of novel

\section{Dovepress}

devices and optimal use of existing devices which will lead to improved clinical outcomes and more effective patient management and safety is a key feature. The manuscript management system is completely online and includes a quick and fair peer-review system. Visit http://www. dovepress.com/testimonials.php to read real quotes from authors. 